\title{
On Effect of Visual Support for Articulation in Pronunciation Correction
}

\author{
Shun Ito, Takafumi Yamazaki, Izumi Hanazaki \\ Dept. of Robotics and Mecatronic, School of Science and technology for Future Life, \\ Tokyo Denki University \\ 5 asahi-cho Senju adachi-ku Tokyo, 120-8551, Japan \\ E-mail: yamazaki_t@hanalab.fr.dendai.ac.jp
}

\begin{abstract}
In this paper, we propose visual support ways for articulation in pronunciation correction and investigate the effect through some experiences. The way which we have proposed is realized by display of formants and shapes of mouth and tongue, which are derived based on the formants of speaker's voice. These displays can lead supported ones to recognize their pronunciation different from correct pronunciation.
\end{abstract}

\section{Introduction}

Speech behavior is one of the skills to learn naturally after being born. The human being detects the difference between sounds that he uttered and ones that he was going to utter and controls his articulator. Its function is called auditory feedback. However, auditory feedback may not be performed well when there is impairment of hearing. In these cases, pronunciation correction where an instructor teach how to control the articulator, are required. The reason is because the speaker cannot detect the difference of the sound and cannot know the way to speak. We aim at the construction of the pronunciation correction system which can play the roll of the instructor based on speech signal analysis. In this paper, we propose visual support for articulation in pronunciation correction and investigate the effect through some experiments about vowel.

\section{Pronunciation Correction System}

\subsection{Outline of Pronunciation Correction System}

In this system, input speech signal is analyzed by AR model and its formants are extracted. Based on the extracted formants, some visual support ways which show the correct manner of articulation to the speaker are constructed. The pronunciation correction system detects the difference between the reference voice and the speaker's voice, and shows visually how to move the tongue and mouth. The 1 st and the 2 nd formants are adopted as acoustic feature quantity of the speech signal which creates the instructions for correct pronunciation.
The system has the following functions.

1) Speech signal analysis

2) Display of formants in normalized formants space between speaker's voice and reference voice

3) Estimation and display of mouth

4) Estimation and display of the shape of the tongue

Each function above will be mentioned in following sessions.

\subsection{Speech Signal Analysis}

The speaker's voice signal is inputted to the system at sampling frequency $10[\mathrm{kHz}]$ and is divided into some frames with length $0.06[\mathrm{~s}]$. The signal of each frame is analyzed using AR (Auto-Regressive) model whose order is 10. AR coefficients are estimated by Burg's method. The power spectrum of the voice signal is extracted based on estimated AR model. The formants are obtained as the frequency at which the power spectrum peak is taken.

\subsection{Display of Formants in Normalized Formants Space between Speaker's Voice and Reference Voice}

Formants of the vowel of persons are different slightly by individual. The reference voices in this system are ones of the Japanese specialist of phonics who can pronounce correctly English vowels. The formant of the target voice which will lead correct pronunciation to the learner is generated on the same formant space of the teacher's voice. We normalize the formants space to remove the difference of both formants.

The voice signal is divided to frames and the formants are estimated from each frame. The $1 \mathrm{st}$, the 2 nd formant of the $i$-th frame are denoted by $F_{1 i}, F_{2 i} . \hat{F}_{1 i}, \hat{F}_{2 i}$ denote normalized formants mapped to the same formant space of the teacher's. It is assumed the $\hat{F}_{1 i}, \hat{F}_{2 i}$ is derived by next equations.

$$
\begin{aligned}
& \hat{F}_{1 i}=\alpha_{12} F_{1 i}{ }^{2}+\alpha_{11} F_{1 i}+\alpha_{10} \\
& \hat{F}_{2 i}=\alpha_{22} F_{2 i}{ }^{2}+\alpha_{21} F_{2 i}+\alpha_{20}
\end{aligned}
$$

where coefficients $\alpha_{m n}(m=1,2 \quad n=0,1,2)$ are decided 
as to minimize $E_{1}, E_{2} . \widetilde{F}_{i 1}, \widetilde{F}_{i 2}$.

$$
\begin{aligned}
& E_{1}=\sum_{i=1}^{N}\left\{\hat{F}_{1 i}-\left(\alpha_{12} F_{1 i}{ }^{2}+\alpha_{11} F_{1 i}+\alpha_{10}\right)\right\}^{2} \\
& E_{2}=\sum_{i=1}^{N}\left\{\hat{F}_{2 i}-\left(\alpha_{22} F_{2 i}{ }^{2}+\alpha_{21} F_{2 i}+\alpha_{20}\right)\right\}^{2}
\end{aligned}
$$

$\widetilde{F}_{i 1}, \widetilde{F}_{i 2}$ means the teacher's formants of the same vowel. In this way, the formants of the learner and of the teacher can be shown in the same formant space formed by the 1st and 2 nd formants. So the learner can confirm the difference between his pronunciation and the target. Display of the formants is shown in Fig. 1.

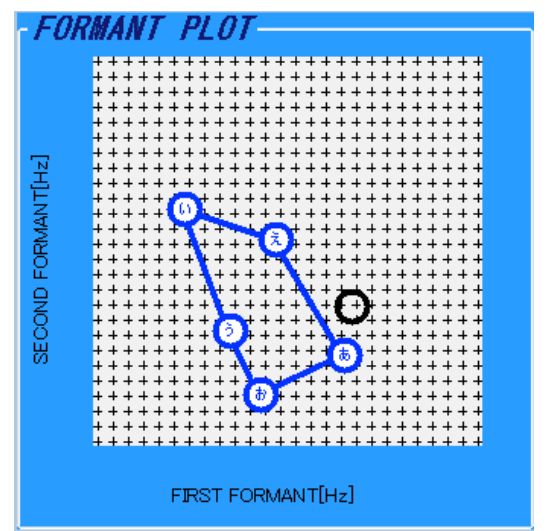

Fig.1 Display of formants

\subsection{Estimation and display of mouth shape}

It is necessary to estimate relations of the mouth open and the formant to generate instructions how to open mouth . Mouth shape is measured at the time of the vowel sound pronunciation by a motion capture in this study. The markers M1, M2, M3, M4 are attached to top / bottom and right /left of lips shown in Fig.2. The vertical difference $d_{1}=\left|M_{1}-M_{2}\right|$ of the mouth and horizontal difference $\quad d_{2}=\left|M_{3}-M_{4}\right|$ are calculated from coordinate $\left\{M_{k}\left(x_{k}, y_{k}, z_{k}\right) ; k=1,2,3,4\right\}$ of markers. $d_{1}(i), d_{2}(i)$ means the mouth shape of the $i$-th frame.$d_{1}(i)$ and $d_{2}(i)$ can relate to the 1 st and the 2 nd formant respectively. We can find out the linear relationship between them. Therefore, the next expression is assumed.

$$
\begin{gathered}
d_{1}(i)=\beta_{11} F_{1 i}+\beta_{10} \\
d_{2}(i)=\beta_{21} F_{2 i}+\beta_{20}
\end{gathered}
$$

where coefficients $\left\{\beta_{m n} ; m=1,2 \quad n=0,1\right\}$ are decided by the least-squares method.

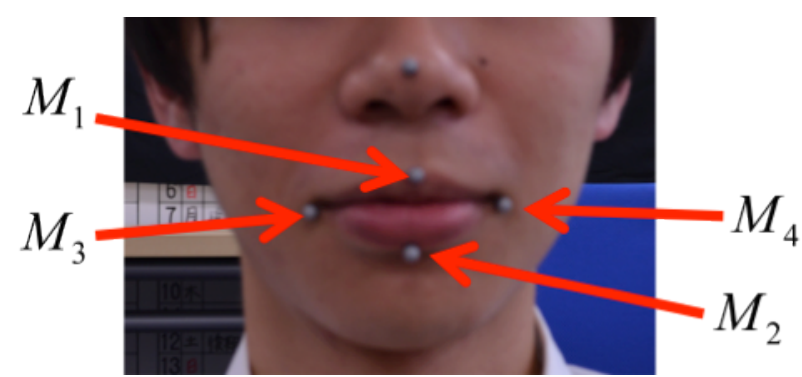

Fig.2 Measurement of the mouth shape by the motion capture

We can calculate respectively the mouth shape and the ideal mouth shape from the formant of the uttered voice and the target voice, and show the instruction immediately.

\subsection{Estimation and display of the shape of the tongue}

We estimate the position of the tongue from formant and use it for visual support for pronunciation correction. The position of the tongue is extracted from image data taken by MRI (magnetic resonance imaging device). MR image taken while uttering the vowel is shown in Fig.3. Because the vocal tract is hollow, it becomes to be black. Estimation of the shape of the tongue are performed in the following processes. The procedure of the estimation is shown in Fig.4. The maxillary and mandibular boundary surfaces of the vocal tract are extracted and the centerline of the vocal tract are calculated. ${ }^{[1]}$ The centerline is divided to the segment with same length. In each segment, the distance between maxillary and mandibular boundary surfaces are measured. The distance are denoted by $T(j)$ and are used as the feature of the tongue shape.

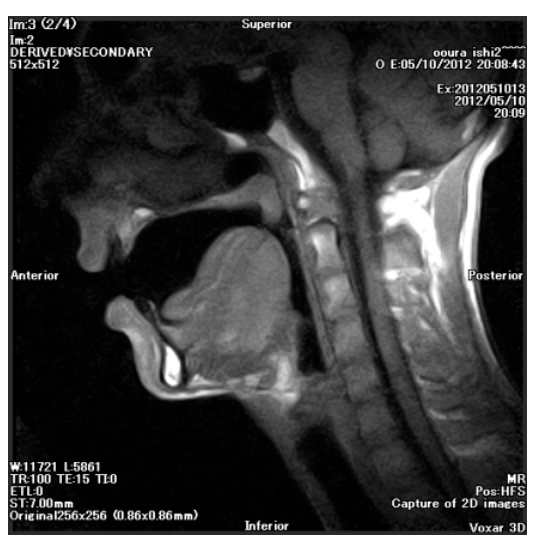

Fig.3 MR image during uttering Japanese vowel 


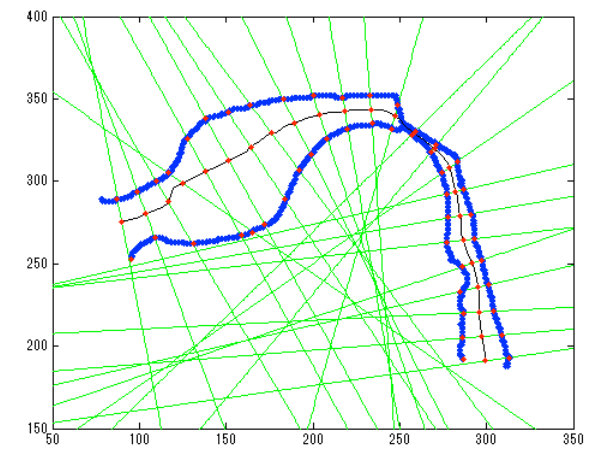

Fig.4 Examples of extracting the position of the tongue

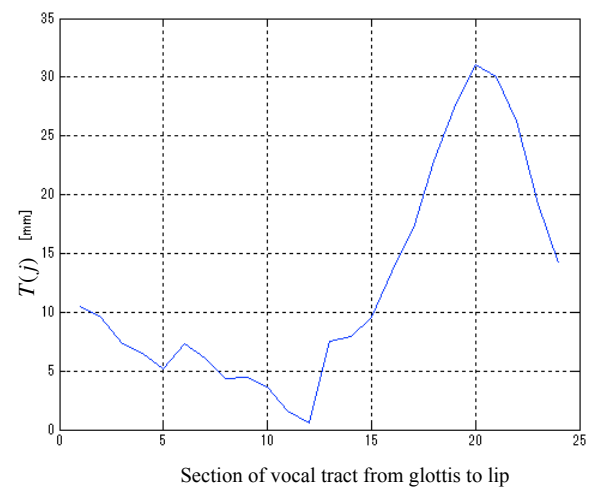

Fig.5 Relations of the shape of a divide and the tongue

In Fig. 5, the feature of the tongue shape is shown. The right-side end of the graph expresses the position of lips. Here, the distance between maxillary and mandibular boundary surfaces, $T(j)$, denotes the tongue shape. $T(j)$ is predicted from the 1 st formant $F_{1}(j)$ and the 2 nd formant $F_{2}(j)$ by the next equation, where $\hat{T}(j)$ is the prediction and $j$ expresses the number of the samples.

$$
\hat{T}(j)=\gamma_{0}+\gamma_{1} F_{1}(j)+\gamma_{2} F_{2}(j)
$$

Here $\left\{\gamma_{0}, \gamma_{1}, \gamma_{2}\right\}$ are regression coefficients. From (7), the shape of each tongue by the formant of a sound and the target sound can be displayed.

\section{Experiment}

In this session, we show some experiments that were performed to inspect the effect of our support system.

\subsection{Experiment Setup}

The subject who is Japanese will practice English pronunciation using our support system. The vowel that is a learning target is an English vowel sound and is a phoneme unlike the Japanese vowel. The sound of the Japanese teacher that accurate English pronunciation specialized in the phonetics was possible was used for the target voice. The vowel [i], [e], and $[\Lambda]$, which are the target English vowels, are shown on the normalized formant space in Fig.6.

The experiment condition is shown in Table1. The subject can look at "formant" and "the shape of the mouth" and "the shape of the tongue" and can hear the target sound. The pronunciation correction is performed by these support ways. The support ways that are available to each subject are shown in table 2. These subjects include the subject who practiced pronunciation while listening to reference voice without the visual aid at all. Practice such this method are commonly used.

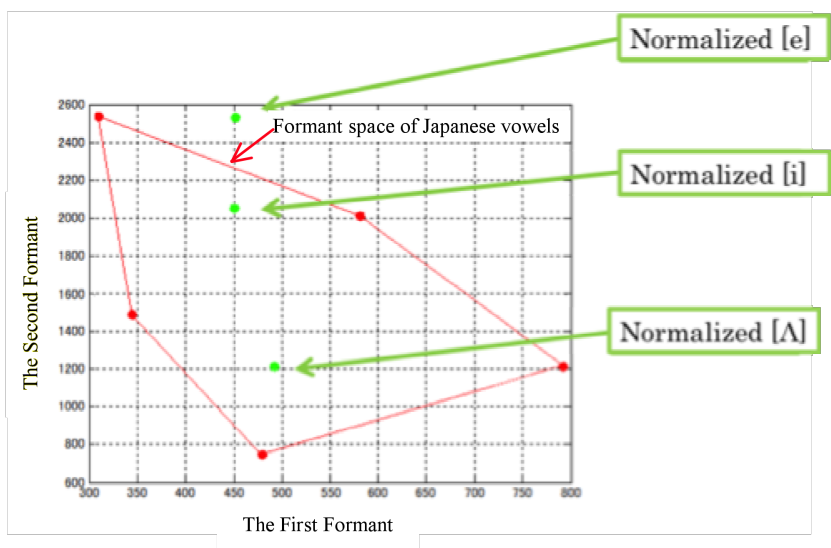

Fig.6 Target vowel voice on the normalized formants space

Table 1 Experiment condition

\begin{tabular}{|c|c|}
\hline Item & Condition \\
\hline Subjects & Nine males \\
\hline $\begin{array}{c}\text { The vowel sound which is } \\
\text { practiced }\end{array}$ & {$[\mathrm{i}],[\mathrm{e}],[\Lambda]$} \\
\hline Exercise period & From July 30 to August 2 \\
& From August 5 to 7 th \\
& From August 20 to $23 \mathrm{rd}$ \\
& August 26 \\
& August 30 \\
\hline Exercise interval & Over two hours \\
\hline
\end{tabular}

Table 2 The support ways that are available to each subject

\begin{tabular}{|c|c|c|c|c|}
\hline Subject & Formants & Mouth & Tongue & Sound \\
\hline Subject 1 & $\bigcirc$ & $\bigcirc$ & $\bigcirc$ & $\times$ \\
\hline Subject 2 & $\bigcirc$ & $\times$ & $\times$ & $\times$ \\
\hline Subject 3 & $\bigcirc$ & $\bigcirc$ & $\times$ & $\times$ \\
\hline Subject 4 & $\bigcirc$ & $\times$ & $\bigcirc$ & $\times$ \\
\hline Subject 5 & $\times$ & $\bigcirc$ & $\times$ & $\bigcirc$ \\
\hline Subject 6 & $\times$ & $\times$ & $\bigcirc$ & $\bigcirc$ \\
\hline Subject 7 & $\times$ & $\bigcirc$ & $\bigcirc$ & $\bigcirc$ \\
\hline Subject 8 & $\times$ & $\times$ & $\times$ & $\bigcirc$ \\
\hline Subject 9 & $\times$ & $\bigcirc$ & $\bigcirc$ & $\times$ \\
\hline
\end{tabular}

$\bigcirc$ :available $\quad x$ : not available 


\subsection{Laboratory finding}

The result of three phases of hearing evaluations by the English teacher is shown in Table3. Utterance motion such that the tongue could go up to the palate were observed in the results attached by '*' in the table 3 . This utterance motion is one of the utterance manner which can be found out in many Japanese. The results of target vowel $[\Lambda]$ were judged not to be right pronunciation, because the uttered sound resembled to the sound [u]. In pronunciation correction for Japanese, the instruction that the sound of [ə] should be pronounced to make a near sound in weak [u]. [ $\Lambda$ ] is strong pronunciation of [ə]. Therefore it is thought that $[\Lambda]$ should be pronounced like $[\mathrm{u}]$ during practice ${ }^{[2]}$.

Table 3 Evaluation of the training sound

\begin{tabular}{|c|c|c|c|}
\hline \multirow{2}{*}{ Subject } & \multicolumn{3}{|c|}{ Training sound } \\
\cline { 2 - 4 } & {$[\mathrm{i}]$} & {$[\mathrm{e}]$} & {$[\Lambda]$} \\
\hline Subject 1 & $\bigcirc$ & $\triangle^{*}$ & $\times$ \\
\hline Subject 2 & $\triangle$ & $\triangle$ & $\times$ \\
\hline Subject 3 & $\bigcirc$ & $\times$ & $\triangle$ \\
\hline Subject 4 & $\times$ & $\triangle$ & $\times$ \\
\hline Subject 5 & $\bigcirc$ & $\times$ & $\times$ \\
\hline Subject 6 & $\triangle$ & $\times$ & $\triangle$ \\
\hline Subject 7 & $\triangle$ & $\bigcirc$ & $\times$ \\
\hline Subject 8 & $\bigcirc$ & $\bigcirc$ & $\triangle$ \\
\hline Subject 9 & $\triangle^{*}$ & $\bigcirc$ & $\triangle$ \\
\hline
\end{tabular}

$\bigcirc:$ Good $\triangle:$ Permission $\times: B a d$

\section{Conclusions}

We can find individual difference in the effect of the visual support in the pronunciation correction from experiments. It must be said the evaluation of the pronunciation correction was not so good. It is because the training period was too short for the learner to achieve correction and to recognize his tongue shape by the instruction of the support system. But we can find some good result in the training stage about a target vowel sound $[\Lambda]$. It may suggest that the proposed way will become effective after some improvements that make the learner be conscious of the shape of the tongue.

\section{References}

[1] Yang Chang Sheng : "Accurate Measurement of Vocal Tract Shapes from Magnetic Resonance Images of Child, Female and Male Subjects", The Journal of the Institute of Electronics, Information and Communication Engineers, SP94-6 pp.1-8 (1994-05)

[2] Sesaburoh Kohmoto: "Aural-Oral Approach to Modern English", Nanundoh, pp44-45, 1978 